\title{
Design and Modeling of an Impulse Continuously Variable Transmission with a Rotational Swashplate
}

\author{
Gang $\mathrm{Li}^{1 *}$ \\ 0000-0003-2793-4615 \\ ${ }^{1}$ Department of Mechanical Engineering, University of Maryland Baltimore County, Baltimore MD, 21250, United States
}

\begin{abstract}
A novel mechanical impulse continuously variable transmission (ICVT) is developed to provide continuously variable speed ratios and smooth acceleration for drivetrains based on a rotational swashplate, and its design principle is illustrated. A swashplate-guide linkage mechanism is used in the ICVT; the speed ratio of the ICVT is changed with the swirl angle of the rotational swashplate. A sliderlinkage system, whose motion is controlled by a speed-regulating handle, is used to adjust the swirl angle of the swashplate. A planetary gear system converts the regulated rotational speed of the swashplate to the output shaft of the ICVT. The speed range of the ICVT can be scaled up by coupling planetary gear systems with different speed ratios. Overrunning clutches are used to rectify the rotational speed from the swashplate-guide linkage mechanism to obtain the output speed of the ICVT. Since the rotational swashplate can introduce large impulse rates of the instantaneous speed ratio, a connecting linkage is used to reduce the impulse rate and unbalanced inertial forces of the ICVT. Experimental tests of the output speed of the ICVT with four guide linkages verify the feasibility of the design and operation performance of the ICVT.
\end{abstract}

Keywords: Continuously variable transmission; Speed ratio; Impulse rate; Kinetic analysis

\author{
* Corresponding author \\ Gang Li \\ gangli@umbc.edu
}

Address: Department of Mechanical Engineering, University of Maryland Baltimore County, Baltimore, MD, United States

Tel: +1 410-455-3309

Fax: +1 410-455-1052

Research Article

Manuscript

$\begin{array}{ll}\text { Received } & 18.09 .2020 \\ \text { Revised } & 08.10 .2020 \\ \text { Accepted } & 06.11 .2020\end{array}$

Doi: 10.30939/ijastech..796723

\section{Introduction}

To improve dynamic performance and fuel efficiency of powertrain systems, some types of automatic transmissions, i.e., infinitely variable transmissions (IVTs), automatic manual transmissions (AMTs), continuously variable transmissions (CVTs), dual clutch transmissions (DCTs), and electrically variable transmissions (EVTs), are used in automotive and wind turbine applications [1-5]. A basic function of automatic transmissions is to smoothly and efficiently convert the torque from a prime mover, such as an engine and an electric motor, to a driven part, such as a wheel and a chain, with a continuous output-to-input speed ratio. Among many different technical issues for developing these automatic transmissions, design of their control systems are crucial to achieve continuous output-to-input speed ratios and superior fuel efficiency [6]. Since AMTs, DCTs, and EVTs use clutch-to-clutch shifts to adjust output-to-input speed ratios, torque interruption occurs during shifts $[7,8]$. Control systems of these clutch-to-clutch shift transmissions are developed by using a combination of a gain-scheduled feedback control and an open-loop control [9]. CVTs enable continuous variation of their speed ratios and torque conversion from a prime mover to a driven part in a wide range of speed ratios to maximize fuel efficiency [9] and reduce transmission errors [10]. While most CVTs convert torques based on friction between pulleys and a belt or a chain, power loss of CVTs is large at the start-up stage of a vehicle when sliding between pulleys and the belt or the chain occurs under a large torque, and the minimum speed ratio of a CVT cannot be zero. Since sliding exists in CVTs, gain-scheduled feedback control and open-loop control are unstable for CVTs [7, 11]. Hence, speed-ratio control is needed for CVTs to maintain a specified speed ratio with optimal clamping forces between pulleys and the belt or the chain for avoiding sliding and maximizing fuel efficiency [12]. In order to improve the capability of large-torque conversion at the start-up stage, some power-split systems are used in combination with traditional CVTs, such as a hydrostatic transmission [13] and a flywheel system [14]. Since coordination of a power-split system and mechanical parts of a CVT cannot be only achieved by me- 


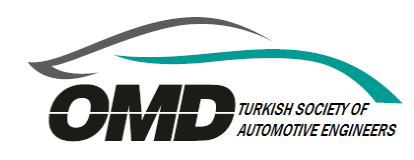

chanical components, a sophisticated electronic control system is inherently needed to optimize power distribution between the CVT and the power-split system $[15,16]$.

The mechanism and the mechanical structure of impulse continuously variable transmissions (ICVTs) are simple and can provide continuously variable speed ratios and reliable power conversion in many applications [17]. Since the speed ratio of the ICVT can be adjusted by the swirl angle of the swashplate, the speed ratio can be zero at the start-up stage. Since the ICVT converts the torque by gear contact forces instead of friction forces of the V-belt of a CVT, the ICVT can transmit a large torque without slippage and the variable range of the speed ratio is larger than that of a CVT. The larger variable range of the speed ratio of the ICVT allows a prime mover to virtually operate in its optimal speed range that is independent of the speed of the driven part to maximize fuel efficiency. Therefore, ICVTs can be widely used in automotive, textile, food and packaging industries [17].

The GUSAII type ICVT was developed with a continuously variable speed ratio [18]. This ICVT has merits of wide speed ratios and simplified output speed control. The output speed of the GUSAII type ICVT can be zero, and the impulse rate of the output speed of the ICVT was optimized. Good manufacturability and reliable transmission performance can be also achieved with its compact structure [18]. An ICVT was developed in [19] with similar characteristics of the GUSAII type ICVT. Since the power range of the ICVT was small, this ICVT can only be used for low torque conditions. Due to reverse handle design, the ICVT can be converted two-direction input speeds to one-direction output speeds. All the above ICVTs [17-19] were designed based on connecting rod transmission mechanism. By changing the length of each rod of a linkage in the ICVT, stepless speed regulation of these ICVTs can be achieved. If a lower pair of the linkage was replaced by a higher pair of cams, connecting rods of the ICVT can be changed to eccentric cam mechanism. The structure of the ICVT with eccentric cam mechanism was much simpler than that of the ICVT with connecting rods. There are also some improved ICVTs besides these major types of ICVTs mentioned above. For instance, a Philamat ICVT was developed based on combination of fixed-axis gear mechanism and connecting rod mechanism [20]. Since the impulse rate of the output speed of the Philamat ICVT was small, the range of its output speed control was wide, and it can be used for high torque conditions. Kazem and Zoltan [21] proposed a novel parallel disc CVT. Kinematic and dynamic characteristics of the parallel disc CVT were analyzed. A MORSE ICVT [22] was designed based on combination of cam mechanism and a gear set.

While structures of the above ICVTs have been improved for multiple applications, there are some shortcomings of ICVTs in practice [23]. First, the inertia force that is generated by the movement of connecting rods is difficult to be eliminated or reduced. Vibration and impacts of the ICVTs caused by the unbalanced inertia force and inertia moment are large when speeds of connecting rods are high. Second, there is no over-speed protection of output shafts of ICVTs. Its bearing capacity and shock resistance is relatively poor and this limits the capacity of transferring power of ICVTs. Third, pulsation of ICVTs cannot be completely eliminated.

This study presents a new ICVT with a rotational swashplate. The transmission component of the ICVT is composed of the rotational swashplate, guide linkages, and a planetary gear set. The proposed ICVT overcomes the major shortcomings of the existing ICVTs with connecting rods or swing links, which have inertia forces or the inertia moments. Since the impulse rate of the output speed of the proposed does not vary with the change of its speed ratio, the impulse rate of the output speed of the proposed ICVT is small.

The remaining part of this paper is organized as follows: the basic layout and design principle of the ICVT is introduced in Sec. 2. Structure of the proposed ICVT with the rotational swashplate are presented in Sec. 3. Some dynamic performance of the proposed ICVT are analyzed in Sec. 4. In order to verify operation performance of the proposed ICVT, some experimental results are discussed in Sec. 5. Finally, some conclusions from this study are presented in Sec. 6.

\section{Layout and Design Principle of the ICVT}

\subsection{Layout and Overview of the ICVT}

The basic principle of the proposed ICVT is to convert the rotational speed of an input shaft to a rotational speed of a swashplate in a swashplate-guide linkage mechanism, and then the rotational speed of the swashplate to the rotational speed of a driving gear in a planetary gear system. The output speed of a sun gear is transmitted by the planetary gear meshing, as shown in Fig. 1. By changing the position of a slider, the swirl angle of the swashplate is changed, which is the angle between the swashplate and the vertical section of the input shaft. If the swirl angle of the swashplate changes, the rotational speed of planetary gears changes, and the amplitude of the output rotational speed of the sun gear changes. Consequently, the speed ratio of the ICVT changes with the swirl angle of the swashplate.

A schematic of the ICVT based on a rotational swashplate is shown in Fig.1. The slider connects to a nut that is installed on the control shaft. The nut is driven by the screw thread. The position of the nut changes with rotation of the control shaft. Hence, the translational motion of the slider can be driven by the rotational motion of the control shaft. The swashplate connects to the input shaft of the ICVT by using a revolute joint at the point $C$. A connecting rod connects the slider and the swashplate at points $A$ and $B$, respectively. The swashplate rotates around the point $C$, and its swirl angle changes with the position of the slider. Rotational motion of the swashplate transmits to planetary gears by using guide linkages. Each planetary gear connects the guide linkage by using an overrunning clutch. The rotational speed of the sun 


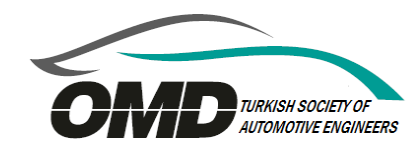

gear is transmitted by gear meshing of the planetary gear system, and it works as the output speed of the ICVT.

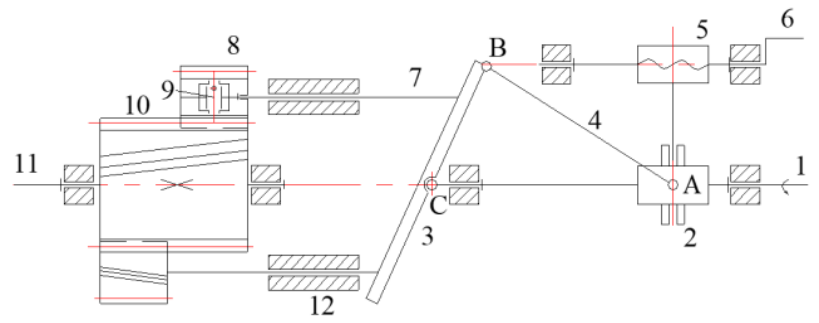

Fig. 1. Layout of the proposed ICVT,

where 1 is the input shaft, 2 is the slider, 3 is the swashplate, 4 is the connecting linkage, 5 is the nut, 6 is the control shaft, 7 is the guide linkage, 8 is the planetary gear, 9 is the overrunning clutch, 10 is the sun gear, 11 is the output shaft, and 12 is the rack.

\subsection{Principle of the ICVT}

The input speed of the ICVT is transmitted from a prime mover to the input shaft of the ICVT. When the input shaft of the ICVT rotates, the power is transmitted to the swashplate with the same rotational speed of the input shaft. Synchronously, the slider and the connecting linkage are also driven by the input shaft with the same rotational speed of the input shaft. The rotational speed of the swashplate is transmitted to the rotational speed of guide linkages by its rotational motion. The rotational speed of the control shaft convert to the translational speed of the nut on the control shaft. The position of the nut is adjusted by the rotational angle of the control shaft. Since the nut connects with the slider by using a rod, the position of the slider can be controlled by the rotational angle of the control shaft. The swirl angle of the swashplate can be controlled by the position of the slider.

Since each planetary gear connects with a guide linkage through the corresponding overrunning clutch, the planetary gear can rotate with the rotational motion of the guide linkage. Rotational motions of guide linkages and planetary gears depend on the rotational speed of the input shaft, the swirl angle of the swashplate, and the installation direction of overrunning clutches. Planetary gears can rotate relative to the corresponding guide linkages when the guide linkages and the planetary gears rotate in the opposite direction due to the one-way rotation property of overrunning clutches.

A pair of the guide linkage and the planetary gear can only achieve the unidirectional intermittent rotation of the sun gear. If the number of the guide linkage is less than three, the motion of the output shaft will be discontinuous. The minimal number of guide linkages is three. The number of guide linkages is four in this study, as shown in Fig.2. If three or more guide linkages and planetary gears are evenly installed on circumferential position along the axis of the sun gear, the sun gear can provide continuously unidirectional rotation of the ICVT.

\section{Structure of the ICVT}

A three-dimensional (3D) structure diagram of the proposed ICVT is shown in Fig. 2. An ICVT mainly includes an inputcontrol module, a motion-conversion module (overrunning clutch), and a speed-control module. The input shaft that is driven by a prime mover serves as the driving part of the ICVT. The rotational swashplate is mounted on the input shaft. The swirl angle of the swashplate is adjusted by the rotational angle of the speed-regulating handle. The swashplate rotates synchronously by the driving of the input shaft. Then the swashplate pushes the guide linkage to move. There are four guide linkages in the 3D structure model, as shown in Fig. 2. Each planetary gears is installed on the corresponding guide linkage. Overrunning clutches are used for over speed protection between the planetary gears and the guide linkages. The rotational speed of four planetary gears convert to the rotational speed of the sun gear of the planetary gear set. The sun gear is installed on the output shaft of the ICVT. Hence, the rotational speed of the output shaft is equal to the rotational speed of the sun gear. Figure 3 shows an experimental prototype of the proposed ICVT.

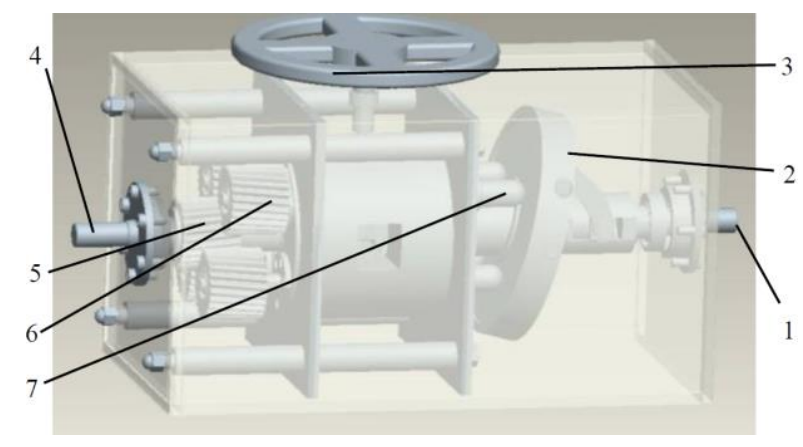

Fig. 2. Structure of the ICVT,

where 1 is input shaft, 2 is the swashplate, 3 is the speed-regulating handle, 4 is the output shaft, 5 is the driven gear, 6 is the driving gear, and 7 is the guide linkage.

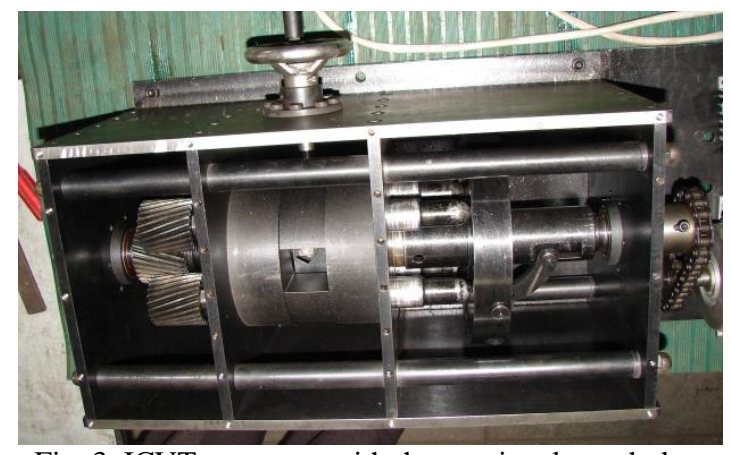

Fig. 3. ICVT prototype with the rotational swashplate

\section{Dynamic Performance of the ICVT with the Rota- tional Swashplate}

The impulse rate of the output shaft of the ICVT decreases with the number of guide linkages increases. Some operation 
performances of the ICVT with different number of guide linkages are analyzed in this section.

\subsection{Rotational Speed of the Output Shaft}

If there is one guide linkage in the ICVT, relation formula between rotational angles of the output shaft and the input shaft can be represented as

$$
\varphi_{u}=\frac{D}{d_{s}} \tan \beta \tan \theta\left(1-\cos \varphi_{p}\right)
$$

where $D$ is the diameter of the distributed circle of guide linkages, $d_{s}$ is the diameter of the pitch circle of the sun gear on the output shaft, $\beta$ is the helical angle of the pitch circle of the sun gear, $\theta$ is the swirl angle of the rotational swashplate, and $\varphi_{p}$ is the rotational angle of the input shaft, i.e., $\varphi_{p}=\int \omega_{p} \mathrm{~d} t$, in which $\omega_{p}$ is the rotational speed of the input shaft, and $t$ is the time. The swirl angle of the swashplate is determined by the length of $\overline{A C}$, which is controlled by the rotational angle of the control shaft.

Since guide linkages alternately work in a rotational period of the input shaft and guide linkages evenly distribute around the revolute joint at the point $C$ on the swashplate, the rotational speed of the output shaft of the ICVT is relative to the number of the guide linkages. The rotational speed of the output shaft of the ICVT can be represented as

$$
\omega_{u i}=\frac{\mathrm{d} \varphi_{u}}{\mathrm{~d} t}=\omega_{p} \frac{D}{d_{s}} \tan \beta \tan \theta \sin \left(\varphi_{p}-\frac{\pi}{2} i\right)
$$

where $i$ is the index of guide linkages, i.e., $i=0,1, \ldots, n-1$, in which $n$ is the number of guide linkages. If there are four guide linkages in the ICVT, one has

$$
i=\left\{\begin{array}{lc}
0 & \pi / 4 \leq \varphi_{p}<3 \pi / 4 \\
1 & 3 \pi / 4 \leq \varphi_{p}<5 \pi / 4 \\
2 & 5 \pi / 4 \leq \varphi_{p}<7 \pi / 4 \\
3 & 0 \leq \varphi_{p}<\pi / 4 \text { or } 7 \pi / 4 \leq \varphi_{p}<2 \pi
\end{array}\right.
$$

Since four overrunning clutches are used here for rotational motion conversion, rotational speeds of the output shaft of the ICVT can also be represented as

$$
\boldsymbol{\omega}_{u i}=\left[\begin{array}{l}
\omega_{u 1} \\
\omega_{u 2} \\
\omega_{u 3} \\
\omega_{u 4}
\end{array}\right]=\omega_{p} \frac{D}{d_{s}} \tan \beta \tan \theta\left[\begin{array}{c}
\sin \varphi_{p} \\
\sin \left(\varphi_{p}-\frac{\pi}{2}\right) \\
\sin \varphi_{p}-\pi \\
\sin \left(\varphi_{p}-\frac{3 \pi}{2}\right)
\end{array}\right]
$$

Since four one-way bearings only allow the maximum rotational speed of four output gears in the clockwise direction to transmit to the output shaft of the IVT, the output speed the IVT can be represented as

$$
\begin{array}{r}
\omega_{u}=\omega_{p} \frac{D}{d_{s}} \tan \beta \tan \theta \max \left\{\sin \varphi_{p}, \sin \left(\varphi_{p}-\frac{\pi}{2}\right),\right. \\
\left.\sin \varphi_{p}-\pi, \sin \left(\varphi_{p}-\frac{3 \pi}{2}\right)\right\}
\end{array}
$$

where $\max \{\cdot\}$ is a function that selects the maximum value of its arguments. Figure 4 shows the rotational speed of the output shaft of the ICVT with four guide linkages.

In Fig. 4 curve above the shadow line is the graph of the angular velocity vs. time where the number of guide linkage is 4 . The point of intersection between the curves is speed intersection between adjacent guide linkages.

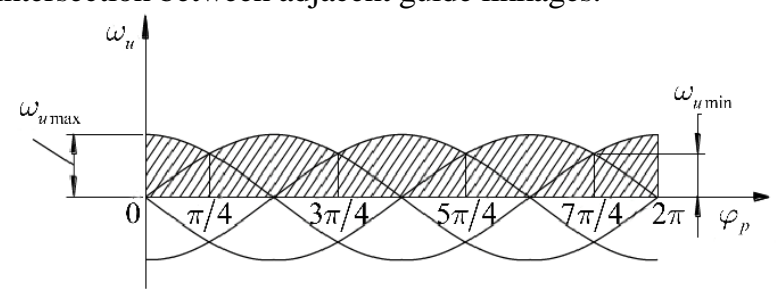

Fig. 4. Rotational speed of the output shaft of the ICVT with four guide linkages

\subsection{Impulse Rate of the ICVT with the Rotational Swash- plate}

While the ICVT can convert variable input speeds to desired output speed with continuously variable speed ratios, variations of output speeds of the ICVT have negative effects on stationarity of its operation performance. The impulse rate is used to evaluate variations of the rotational speed of the output shaft of the ICVT.

According to Eq. (5), the maximum rotational speed of the output shaft of the ICVT does not change with changes of the number of guide linkages. The maximum rotational speed of the output shaft of the ICVT can be represented as

$$
\omega_{u \max }=\omega_{p} \frac{D \tan \beta \tan \theta}{d_{s}}
$$

The minimum rotational speed of the output shaft of the ICVT occurs at the intersection angle between two adjacent guide linkages that can be represented as

$$
\omega_{u \min }=\omega_{p} \frac{D \tan \beta \tan \theta}{d_{s}} \sin \left(\frac{\pi}{n} i+1\right)
$$

where $n$ is the number of the guide linkages. According to Eq. (7), the minimum rotational speed of the output shaft of the ICVT changes with changes of the number of guide linkages. The impulse rate of the ICVT can be represented as

$$
\delta=\frac{\omega_{u \max }-\omega_{u \min }}{\bar{\omega}_{u}}
$$

where $\bar{\omega}_{u}$ is the average rotational speed of the output shaft of the ICVT that can be represented as

$$
\bar{\omega}_{u}=\frac{1}{2 \pi} \int_{0}^{2 \pi} \omega_{p} \frac{D}{d_{s}} \tan \beta \tan \theta \sin \left(\varphi_{p}-\frac{\pi}{2} i\right) \mathrm{d} \varphi_{p}
$$




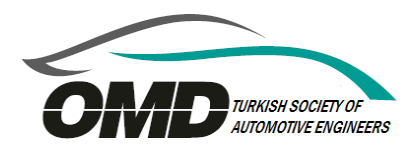

According to Eq. (8), the minimum rotational velocity changes with the number of the guide linkages of the ICVT. The impulse rate of the ICVT depends on the maximum and the minimum rotational speed of the output shaft of the ICVT. Based on Eq. (7), the minimum rotational speed of the output shaft of the ICVT can be decreased with the increment of the number of guide linkages.

\subsection{Continuously Variable Speed Ratio of the ICVT with the Rotational Swashplate}

The average speed ratio of the proposed ICVT can be represented as

$$
\bar{\lambda}_{n}=\frac{\bar{\omega}_{p}}{\bar{\omega}_{u}}=\frac{2 \pi}{n \Delta \varphi_{u}}
$$

where $n$ is the number of guide linkages, $\bar{\omega}_{p}$ is the average rotational speed of the input shaft, $\bar{\omega}_{u}$ is the average rotational speed of the output shaft, and $\Delta \varphi_{u}$ is the corresponding rotational angle of the output shaft when the input shaft rotates an angle of $2 \pi / n$.

The speed ratio of the ICVT is defined as $\lambda_{n}=\omega_{p} / \omega_{u}$. Based on Eq. (6), the speed ratio of the ICVT can be represented as

$$
\lambda_{n}=\frac{d_{s}}{D \tan \beta \tan \theta \sin \left(\varphi_{p}-\frac{\pi}{2} i\right)}
$$

Since the minimum rotational velocity of the output shaft can be zero, variable speed ratio of the ICVT with the rotational swashplate is infinity. The variable speed ratio of the ICVT with the rotational swashplate is irrelevant to the number of guide linkages. Characteristic parameters of the ICVTs, which have different numbers of guide linkages, can be obtained by Eqs. (8)-(11).

Based on Eqs. (8) and (9), the impulse rate of the ICVT with the rotational swashplate is significantly reduced with increasing the number of guide linkages. While the total weight of the ICVT is slightly increased with more guide linkages under same other design conditions, the volume of ICVTs with different number of guide linkages is the same. If the number of guide linkages is six, the impulse rate of the ICVT obviously decreases compared with other ICVTs with fewer guide linkages. Additionally, impulse rates of ICVTs with different number of guide linkages are irrelevant to their speed ratios.

\section{Experimental Results and Discussion}

The ICVT system as it is during experimental tests is shown in Fig. 3. Experimental tests for the operation performance of the ICVT prototype with four guide linkages are conducted on a test rig. This experimental setup of the ICVT system consists of an ICVT, a permanent magnet DC motor, a magnetic brake, a torques sensor, and an angular encoder. The DC motor that is used as the prime mover of the ICVT system is connected with the input shaft of the ICVT. The magnetic brake mounted on the output shaft is used to provide a variable load for the ICVT system. The angular encoder is mounted on the output shaft of the ICVT to measure its rotational angles.

Gear pairs and bearings of the ICVT are lubricated by Gear Oil VG100 during tests to provide favorable lubrication conditions to minimize friction of meshing gear tooth surfaces [24]. The DC motor and the magnetic brake are both mounted on rigid pedestals, and connected with the corresponding shafts by couplings to eliminate their eccentric effects. The ICVT system was initially tested for $4 \times 10^{4}$ input cycles with an input load of 40 $\mathrm{Nm}$ and a input speed $100 \mathrm{rpm}$ to assess adhesion of coating of gear tooth surfaces in the running-in period.

Table 1. Design parameters of the ICVT prototype

\begin{tabular}{c|c}
\hline Item & Value \\
\hline $\begin{array}{c}\text { Rotational speed of the input shaft (mm) } \\
\text { ages (mm) }\end{array}$ & 100 \\
\hline $\begin{array}{c}\text { Diameter of the distributed circle of guide link- } \\
\text { (deg) }\end{array}$ & 108 \\
\hline $\begin{array}{c}\text { Pitcal angle of the pitch circle of the sun gear } \\
\text { Pitch diameter of planetary gears (mm) }\end{array}$ & 15 \\
\hline Number of guide linkages & 48 \\
\hline
\end{tabular}

Table 2. Impulse rate with different swirl angles of the swashplate

\begin{tabular}{c|c|c|c|c}
\hline Item & \multicolumn{4}{|c}{ Value } \\
\hline $\begin{array}{c}\text { Swirl angle of swashplate } \\
\text { (deg) }\end{array}$ & 2 & 5 & 10 & 15 \\
\hline Impulse rate & 0.351 & 0.352 & 0.348 & 0.349 \\
\hline
\end{tabular}

Experimental tests for constant forward speeds are performed to validate the control strategy with constant input-to-output speed ratios. The rotational speed of the output shaft of the ICVT prototype that has four guide linkages was tested, and test conditions of the testing are listed in Table 1. According to Eq. (10), the speed ratio of the ICVT changes with the swirl angle of the swashplate $\theta$. During tests of the impulse rate of the ICVT, the swirl angle of the swashplate, in turn, was set to $2 \mathrm{deg}, 5 \mathrm{deg}, 10$ $\mathrm{deg}$, and $15 \mathrm{deg}$. Testing results of the impulse rate of the ICVT with different swirl angles of the swashplate $\theta$ are shown in Table 2. Based on Eq. (8), the theoretical impulse rate of the ICVT is 0.343 . Testing results of the impulse rate of the ICVT with different swirl angles of the swashplate are larger than the theoretical impulse rate. In these testing results in Table 2, the maximum impulse rate of the ICVT is 0.352 , and occurs when the swirl angles of the swashplate is $5 \mathrm{deg}$. The maximum relative error between the theoretical and testing results of the impulse rate is $2.6 \%$. Errors between the theoretical and testing results of the impulse rate mainly caused by clearance of rollers, manufacturing errors, and misalignments of gear pairs. 


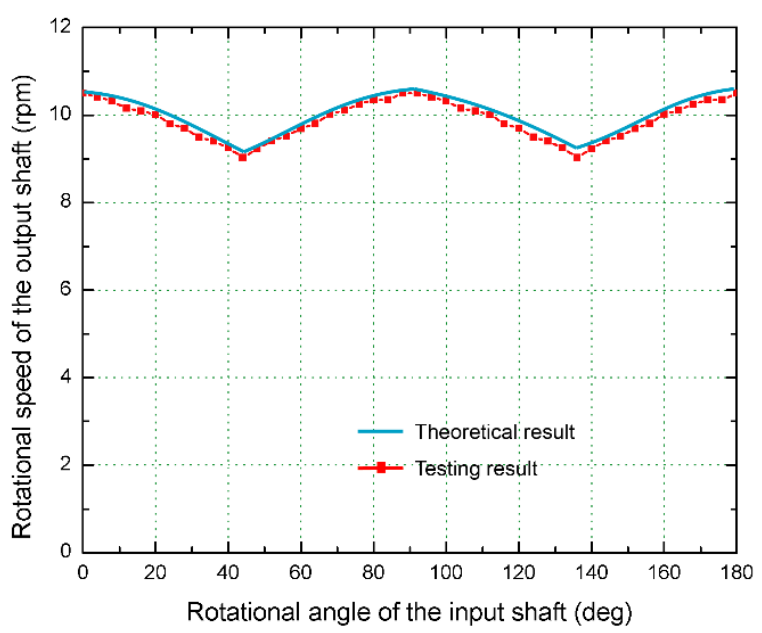

Fig. 5 Rotational speeds of the output shaft of the ICVT with four guide linkages

Testing results of the rotational speed of the output shaft of the ICVT is shown in Fig. 5. During tests of the rotational speed of the output shaft of the ICVT, the swirl angle of the swashplate is set to $10 \mathrm{deg}$. Based on testing results in Fig. 5, errors between the theoretical and testing results of the rotational speed of the output shaft are small. Since rollers in overrunning clutches have some clearance, testing results of the rotational speed of the output shaft are always smaller than theoretical results. The maximum error between the theoretical and testing results of the rotational speed of the output shaft is $0.46 \mathrm{rpm}$, and it occurs when the input angle of the ICVT is $136.47 \mathrm{deg}$. The maximum relative error the theoretical and testing results of the rotational speed of the output shaft is $4.87 \%$. The minimum relative error between the theoretical and testing results of the rotational speed of the output shaft of the ICVT is $1.63 \%$. Their average relative error of the rotational speed of the output shaft of the ICVT is $2.41 \%$. Speed fluctuations of the output speed of the ICVT are caused by the impulse rate of the ICVT, as shown in Fig.5. As mentioned above, the impulse rate of the ICVT can be decreased with the increment of the number of guide linkages.

\section{Conclusions}

This work presents a new ICVT with a rotational swashplate to mechanically transmit power with a continuously variable speed ratio. The proposed ICVT involves of a swashplate-guide linkage mechanism and a parallel-shaft planetary gear system. Speed ratios and impulse rates of ICVTs with different number of guide linkages are analyzed by motion equations. Experimental tests for output speed of the ICVT with four guide linkages are conducted to verify the feasibility the design principle of the proposed ICVT with the rotational swashplate. Based on numerical and testing re- sults obtained, some meaningful conclusions are drawn below.

(1) The proposed ICVT can in principle be used for converting variable input speeds to a desired constant output speed with continuously variable speed ratios.

(2) Uneven impulse rate of the ICVT is irrelevant to its speed ratio. This feature of the ICVT overcomes the shortcoming that impulse rate dramatically changes with low rotational speeds of the output shaft.

(3) The impulse rate of the ICVT with the rotational swashplate is significantly reduced with increasing the number of guide linkages. Theoretically, the impulse rate of the ICVT can be eliminated.

\section{Acknowledgment}

The author is grateful for the financial support from the Maryland Energy Innovation Institute Energy Innovation Seed Grant.

\section{Nomenclature}

D : Diameter of the distributed circle of guide li nkages $(\mathrm{mm})$

$d_{s} \quad$ : Diameter of the base circle of the sun gear (mm)

$\beta \quad$ : Helical angle of the base circle of the sun $g$ ear (deg)

$\theta \quad:$ Swirl angle of the rotational swashplate (deg)

$\varphi_{p} \quad$ : Rotational angle of the input shaft (deg)

$\omega_{p} \quad$ : Rotational speed of the input shaft (rpm)

$t \quad$ : Time (s)

$i \quad:$ Index of guide linkages

$n \quad:$ Number of guide linkages

$\bar{\omega}_{p} \quad$ : Average rotational speed of the input shaft (r pm)

$\bar{\omega}_{u} \quad$ : Average rotational speed of the output shaft (rpm)

$\omega_{u \max }:$ Maximum rotational speed of the output shaft (rpm)

$\omega_{u \min }:$ Minimum rotational speed of the output shaft (rpm)

$\lambda_{n} \quad:$ Speed ratio

$\bar{\lambda}_{n} \quad:$ Average speed ratio

\section{References}

[1] Wang, X. F., and Zhu, W. D. (2018). Design and stability analysis of an integral time-delay feedback control combined with an open-loop control for an infinitely variable transmission system. ASME Journal of Dynamic Systems, Measurement, and Control, 140(1), p. 011007.

[2] Wang, X. F., and Zhu, W. D. (2016). Design, modeling, and 
experimental validation of a novel infinitely variable transmission based on scotch yoke systems. ASME Journal of Mechanical Design, 138(1), p. 015001.

[3] Ross, M. (1997). Fuel efficiency and the physics of automobiles. Contemporary Physics, 38(6), 381-394.

[4] Manwell, J. F., McGowan, J. G., and Rogers, A. L. (2010). Wind Energy Explained: Theory, Design and Application, John Wiley \& Sons.

[5] Yan, J., Li, G., and Liu K. (2020). Development trend of wind power technology. International Journal of Advanced Engineering Research and Science, 7(6), 124-132.

[6] Rupp, D., and Guzzella, L. (2010). Adaptive internal model control with application to fueling control. Control Engineering Practice, 18(8), 873-881.

[7] Sun, Z. X., and Hebbale, K. (2005). Challenges and opportunities in automotive transmission control. Proceedings of the 2005 American Control Conference, Portland, 3284-3289.

[8] Kulkarni, M., Shim, T., and Zhang, Y. (2007). Shift dynamics and control of dual-clutch transmissions. Mechanism and Machine Theory, 42(2), 168-182.

[9] Srivastava, N., and Haque, I. (2009). A review on belt and chain continuously variable transmissions (CVT): Dynamics and control. Mechanism and Machine Theory, 44(1), 19-41.

[10]Hu, Y. H., Li, G., Zhu, W.D., and Cui, J. K. An elastic transmission error compensation method for rotary vector speed reducers based on error sensitivity analysis. Applied Sciences, 10(2), 481.

[11]Pner, R., Guzzella, L., and Onder, C. (2003). Fuel-optimal control of CVT powertrains. Control Engineering Practice, 11(3), 329-336.

[12]Zhu, C., Liu, H., Tian, J., Xiao, Q., and Du, X. (2010). Experimental investigation on the efficiency of the pulley-drive CVT. International Journal of Automotive Technology, 11(2), 257261.

[13]Savaresi, S. M., Taroni, F. L., Previdi, F., and Bittanti, S. (2004). Control system design on a power-split CVT for highpower agricultural tractors. IEEE/ASME Transactions on Mechatronics, 9(3), 569-579.

[14]van Berkel, K., Hofman, T., Vroemen, B., and Steinbuch, M. (2012). Optimal control of a mechanical hybrid powertrain. IEEE Transactions on Vehicular Technology, 61(2), 485-497.

[15]Pesgens, M., Vroemen, B., Stouten, B., Veldpaus, F., and Steinbuch, M. (2006). Control of a hydraulically actuated continuously variable transmission. Vehicle System Dynamics, 44(5), 387-406.

[16]Setlur, P., Wagner, J. R., Dawson, D. M., and Samuels, B. (2003). Nonlinear control of a continuously variable transmission (CVT). IEEE Transactions on Control Systems Technology, 11(1), 101-108.

[17]Ruan, Z. T. (1999). Design and Selecting Guidelines of Mechanical Continuous Variable Transmission. Beijing: Chemical Industry Press.

[18]GUSA. (1988). Variable Speed Gear Drive. Heinrich Gensheimer \& Sohne co.
[19]Zhou, Y. Q. (2001). Mechanical Continuous Variable Transmission. Beijing: China Machine Press.

[20]Zhou, Y. Q., Cui, X. L., and Dong, Z. F. (2005). Introduction and development of mechanical variable speed drives. Journal of Mechanical Transmission, 29 (1): 65-68.

[21]Kazem, K., and Zoltan, F. S. (2005). Parallel disk continuously variable transmission (PDCVT). Mechanism and Machine Theory, 40 (9): 1-30.

[22]Ruan, Z. T. (1999). Mechanical Continuous Variable Transmission. Beijing: China Machine Press.

[23]Zhu, Y., and Liu, K. C. (2003). The present situation of research and development of impulse stepless speed variator. Packaging and Food Machinery, 21 (5): 11-14.

[24]Li, G., Wang, Z. H., and Zhu, W. D. (2019). Prediction of surface wear of involute gears based on a modified fractal method. ASME Journal of Tribology, 141(3), 031603. 